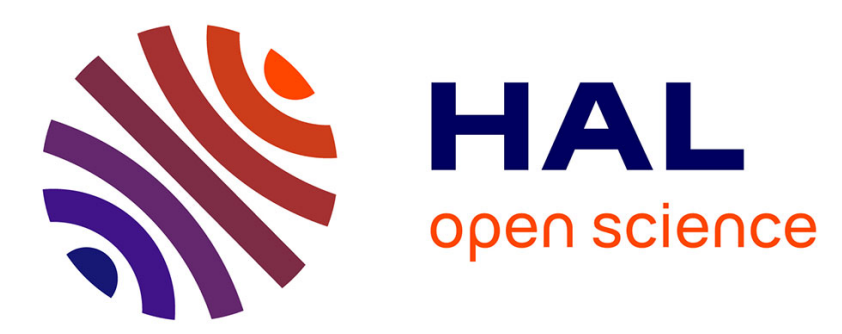

\title{
Quantifying CD95/cl-CD95L Implications in Cell Mechanics and Membrane Tension by Atomic Force Microscopy Based Force Measurements
}

Anaïs Sadoun, Pierre-Henri Puech

\section{- To cite this version:}

Anaïs Sadoun, Pierre-Henri Puech. Quantifying CD95/cl-CD95L Implications in Cell Mechanics and Membrane Tension by Atomic Force Microscopy Based Force Measurements. Patrick Legembre. CD95 Methods and Protocols, 70, Springer, pp.139-151, 2017, 978-1-4939-6778-0. 10.1007/978-1-4939-67803_14. hal-01530854

\section{HAL Id: hal-01530854 \\ https://hal.science/hal-01530854}

Submitted on 31 May 2017

HAL is a multi-disciplinary open access archive for the deposit and dissemination of scientific research documents, whether they are published or not. The documents may come from teaching and research institutions in France or abroad, or from public or private research centers.
L'archive ouverte pluridisciplinaire HAL, est destinée au dépôt et à la diffusion de documents scientifiques de niveau recherche, publiés ou non, émanant des établissements d'enseignement et de recherche français ou étrangers, des laboratoires publics ou privés. 


\section{Quantifying CD95/cl-CD95L implications in cell mechanics \& membrane tension by atomic force microscopy based force measurements}

Anaïs Sadoun, Pierre-Henri Puech*

${ }^{1}$ Aix Marseille Université, LAI UM 61, Marseille, F-13288, France.

${ }^{2}$ Inserm, UMR_S 1067, Marseille, F-13288, France.

${ }^{3}$ CNRS, UMR 7333, Marseille, F-13288, France.

* Email : pierre-henri.puech@inserm.fr

\section{Summary}

Atomic Force Microscopy (AFM) is an invaluable tool to investigate the structure of biological material surfaces by imaging them at nanometer scale in physiological conditions. It can also be used to measure the forces and mechanics from single molecule interaction to cell / cell adhesion. Here, we present a methodology that allows to quantify cell elastic properties (using the Young modulus) and cell membrane tension modulated by CD95/cl-CD95L interactions by coupling nanoindentation and membrane tube pulling using suitably decorated AFM levers.

\section{Keywords}

Atomic Force Microscopy, elasticity, Young modulus, membrane tubes, membrane tension, cytoskeleton. 


\section{Introduction}

CD95L (FasL) is a potent apoptosis inducing factor through its ligation to CD95 (Fas) and has been implicated in carcinogenesis ${ }^{1}$. After cleavage via metalloproteases, CD95L (designated cl-CD95L for cleaved-CD95L) is no more leading to cell death but has been shown to induce migration phenotype in cancer cells and activated $\mathrm{T}$ cells ${ }^{2}$. Recently, this cleaved ligand was shown to augment plasma membrane fluidity ${ }^{3}$, similarly to what happens during epithelial to mesenchymal transition (EMT), and is an intrumental step in allowing cell migration. Such a physical parameter of cell membrane has been linked to prognosis of cancer ${ }^{4}$. Several characterisations of membrane physical properties, such as diffusive capabilities linked to their structure ${ }^{5}$ or mechanical properties such as their elasticity ${ }^{6}$ or their tension ${ }^{7}$ have been performed over the last decades and have been linked to crucial modifications of cell function, such as trafficking, cell spreading or migration ${ }^{8}$. Recently, cell tension has been elegantly studied in the context of EMT ${ }^{9}$. To our knowledge, characterizing membrane tension in relation with CD95/Cl-CD95L has never been performed. We propose here a protocol using Atomic Force Microscopy in force mode that allows us to measure cell elasticity and membrane tension, while stimulating cells with cl-CD95L.

The atomic force microscope (AFM), coming from a material science and physics background, was invented in the $80^{\prime} \mathrm{s}^{10}$, is a member of the scanning probe family of microscopes. It has found use in biology by allowing biologists to image the topography of biological surfaces with nanometer vertical and lateral resolution, using a sharp tip at the end of a soft cantilever to delineate the surface. This high resolution imaging in three dimensions requires little or no treatment (fixation is not mandatory) and can be performed under physiologically-relevant conditions (in liquid medium, at $37^{\circ} \mathrm{C}$ ) ${ }^{11}$. In addition, AFM - force mode (so called « force spectroscopy ») has been developed over the years as a sensitive force measuring technique, able to detect or exert forces in the $\mathrm{pN}$ to $\mathrm{nN}$ range ${ }^{12,13}$, the soft lever being used as a spring which deformation reports the forces exerted at its extremity.

To probe cell mechanics, at single cell scale, several techniques have been developped to monitor either the local or the global mechanics over the last decades, such as micropipette aspiration ${ }^{14}$ and indentation ${ }^{15}$, optical tweezers ${ }^{8}$, microplaques ${ }^{16}$ and AFM. AFM has proved to be one of the most versatile techniques, allowing to obtain mechanical data from pressing on the biological substrate, either locally ${ }^{17}$ or globally ${ }^{18}$, and from pulling on the cell's membrane ${ }^{19,20}$. Several variants have been proposed, allowing to dissect viscoelastic regimes of cell mechanics ${ }^{21,22}$ and even recently to couple 
mechanical measurements with topographical imaging ${ }^{23}$

We describe here a methodology which enables to measure modulations of living cell mechanical properties coming from the interaction between CD95 and cl-CD95L, and to do this at two levels : on both the elastic behaviour, via their Young modulus, and their membrane tension. This uses AFM force mode with decorated, "sticky" levers. Pressing a lever which tip has a known shape allows to measure the Young modulus of the cell (the lower the softer the sample) ${ }^{24}$; pulling the very same lever eventually allows the extraction of a tube, and the force needed to extract it is related to the local tension of the cell membrane ${ }^{9,20}$. Discussion about data processing is also presented. 


\section{Chemicals and biological reactives}

- 4-(2-hydroxyethyl)-1-piperazineethanesulfonic acid (HEPES) (Carl Roth GmbH 9105.3)

- Concanavalin A (Sigma, \#C2272)

- DMEM (Gibco) 41966-029

- Fetal bovine serum (FBS) (Gibco, \#10270)

- Geneticin (Gibco, \#10131)

- Helmanex (Helma)

- L-glutamine (Gibco, \#25030)

- Phosphate-buffered saline (PBS) without Ca2+, Mg2+ (Gibco, \#14190094)

- Puromycin (Sigma): P8833-10mg

- Trypsin-EDTA (0.5\%) (Gibco, \#25300), alternatively Accutase (PAA L11-007)

\section{Material}

- Atomic Force Microscope, preferentially coupled to an optical microscope ${ }^{\mathrm{i}}$

- AFM levers (MLCT-UC, Brucker Instruments or other soft ( $k<60 \mathrm{pN} / \mathrm{nm}$ preferentially) ones $\left.{ }^{\mathrm{ii}}\right)$

- Cell culture pipettes and flasks

- Glass bottom petri dishes (WPI Instruments, Fluorodish FD-100)

- MilliQ water (Millipore)

- Micropipette systems (Gibson or else)

- Steritop filter units (500 ml) (Millipore, \#SCGP U01 RE)

- Vacuum grease 


\section{Procedures}

\section{Cell culture}

Cell culture medium : Prepare sterile DMEM 8\% FBS 2mM L-glutamine. Store at $4^{\circ} \mathrm{C}$ and let warm gently at room temperature before use under the culture hood or for AFM experiments.

Cells lines : Epithelial cell lines MDA-MB-231 (ATCC, ${ }^{25}$ ) and BT 549 (ATCC, ${ }^{26}$ ) were obtained from P. Legembre's lab (Rennes, France) as cultures sent by DHL delivery system. On reception, medium was removed and fresh complete medium added. Cells were let to recover for at least one day before being passaged for the first time. Usually, only the third passage was used for the first experiments. Protocols were adapted to fit the closest possible the ones from P. Legembre's lab.

Cell passaging: the generation time is around 48hrs and are passaged 3 times a week using Trypsin/EDTA (1 min@ @37 C ; quenched with FBS supplemented medium ; centrifugation 5min @ 1000 rpm @ RT), with 80 \% confluent cell layers as assessed by microscopic observation ${ }^{\mathrm{iii}}$.

\section{Cell deposition on glass bottom petri dish}

The goal is to have well adherent, spread and separated cells to be able to test as many single cells in a sample as possible over the time course of the experiment (typically 1hr) before replacement of the sample and/or lever.

The day before the experiment :

1. Recover cells from culture flasks by using short ( 1min @ 37 C) Trypsin / EDTA treatment, quenching Trypsin with FBS supplemented medium, centrifugation (1000 rpm, 5 min, RT) and dilution in culture medium.

2. Seed a small volume of cells (typically 250 $\mu \mathrm{L}$ ) the day before the experiment, in culture medium, on sterile glass bottom 35mm petri dishes filled with $3 \mathrm{~mL}$ of culture medium. ${ }^{\text {iv }}$

3. Let the cells adhere and spread over night at $37^{\circ} \mathrm{C}$ under controlled $\mathrm{CO}_{2}$ atmosphere

The day of the experiment :

1. Assess spreading and density of the cells by bright field microscopy.

2. Prior to experiment, rince the petri dish three times with fresh medium, at ambient temperature 
to minimize thermal adaptation of the AFM lever and remove unbound cells and floating debris if any.

3. Mount the Petri dish in a proper holder (here, the JPK Petri dish Holder) and set, if needed, the temperature to the one needed ${ }^{\mathrm{v}}$. To increase mechanical stability, use thin layers of vacuum grease to ensure a tight fitting of the Petri dish to the holder.

4. Install the sample onto the microscope. Focus on the cells and find a place where cells are numerous, but also well separated, with a bare zone where the lever calibration will be performed (see below).

\section{Cantilever functionalization}

Following published protocols, clean, gold less with pyramidal tip cantilevers ${ }^{\mathrm{vi}}$ were functionnalized by adsorbing a lectin onto $\mathrm{it}^{\mathrm{vii}}$.

1. Clean cantilevers were activated by a 1 min exposure to residual air plasma.

2. Dip them immediately in a solution of either conA or WGA for at least an hourviii

3. Gently rince them by agitation in PBS1x without $\mathrm{Ca}++/ \mathrm{Mg}++$.

4. Use them immediately or store them in sterile PBS, at $4^{\circ} \mathrm{C}$ for maximum 3-4 days.

\section{Cantilever mounting, calibrating and setting}

This part is dependent on the precise AFM hardware used. The present directions are for JPK Instruments NanoWizard.

First, prepare the lever and its holder.

1. Clean the glass block (cantilever holder) by gently rubbing it with a Q-tip soaked in Helmanex / water solution

2. Rince it by flushing ethanol and MQ water alternaltively, at least three times, and finishing by water. Dry it rapidly and carefully under a stream of azote or air.

3. Mount the cantilever, keeping it wet, and adjust the fixation so that it will be held strongly on the glass block ${ }^{\mathrm{ix}}$. 
4. Set the holder onto the AFM head or module.

Second, set the sample in place (see section Measurements below) and rapidely process to the first steps that follow.

1. Introduce the lever / holder into the sample and check that it is properly dipping into the medium.

2. Focus the laser onto the lever's back, eg. using the inverted microscope's camera ${ }^{x}$ and set it to have the maximal sum signal on the QPD.

3. Let the system reach a thermal equilibrium for 5 to $10 \mathrm{~min}$, depending on the temperature difference between the room and the medium.

4. Engage, with a moderate force, the lever onto a bare zone of the sample, and take several force curves with a relatively high setpoint in order to get a linear curve in the contact region. ${ }^{\text {xi }}$

5. Fit the contact region with a line to get the sensitivity of the set-up. Average over at least three independent force curves.

6. Retract the lever at least $100 \mu \mathrm{m}$ from the surface and record the oscillations of the lever under brownian motion. Analyse the spectrum to get the spring constant following ${ }^{30}$

7. Set the force measurement parameters : maximal pressing force, force curve length, speed of pressing and pulling, contact and recovery times, acquisition frequency. Typical values are, respectively, $100-250 \mathrm{pN}, 5 \mu \mathrm{m}, 2 \mu \mathrm{m} / \mathrm{s}, 2 \mu \mathrm{m} / \mathrm{s}, 0.1-1 \mathrm{~s}, 0-5 \mathrm{~s}, 2048 \mathrm{~Hz}$. Increasing the contact time and the contact force are intuitively linked to the increase in the probability of having binding, hence to be able to extract a tube ${ }^{\text {xii }}$. Set them to the desired values.

\section{Measurements}

1. Incubate the sample either with cl-CD95L (100ng/mL final concentration) or control molecule (eg. pcDNA3, at same dilution), or carrier medium for at least $30 \mathrm{~min}$ before starting the experiment. Exchange the medium for perturbator free medium if needed. Then proceed to the introduction of the lever / holder in the sample (see above). ${ }^{\text {xii }}$

2. Choose a cell and bring it close to the lever position.

3. Capture a video micrograph of the cell of interest 
4. Re-engage the lever before moving it above the desired cell or cell part (nucleus, lamellipodia see Fig. 1).

5. Record several force curves per cell, before moving the lever away from the cell. The crucial parameters to keep constant and/or adjust are the contact time, the contact force and the speeds of pressing and pulling ${ }^{\text {xiv }}$.

6. Capture a second video micrograph of the cell of interest to document any morphological change during the experiment.

7. Repeat steps 1-7 up to 25 cells or 1 h30 (typically) of experiment, before changing sample and/lever ${ }^{\mathrm{xv}}$

At this stage, two possibilities exist for comparing conditions : (A) separate experiments, with control conditions and desired sample; (B) coupled experiments, with the same sample, with added soluble perturbation (here cl-CD95L). In the case (A), repeat the previous procedure with every sample. The case (B) is detailled below.

1. Start the experiment following the above procedure with an unperturbed sample.

2. Perform measurements on a limited set of cells for 45 min

3. Remove the head of the AFM or use sample holder side ports to introduce cl-CD95L (or control condition, eg. control pcDNA) to the desired final concentration, and wait several minutes to let it diffuse to uniform concentration above the sample.

4. Start again the measurements, either taking the first cells at hand or trying to find back the very same cells that were used in step 1. Each way will have profound impact on the statistical way to process the data and on the interpretation of the data.

A variant of (B) allows theoretically to have a kinetics, on a single cell, of the perturbation. For this, a high frequency of tube pulling may be required, increasing per se the risks of damaging the cell and/or introducing artifacts.

If needed (see below), to obtain data decoupling the membrane from the cytoskeleton, experiments have to be reproduced with samples pre-treated with small amounts of Latrunculin A following 19,20

\section{A short description on data processing}


From the force curves, as shown on Fig. 1D, E, one can extract two physical parameters.

\section{Young modulus}

From the pushing part of the curve, the Young modulus ( $E$, in Pa) that represent the elasticity of the cell

(the stiffer the cell, the higher its value) can be extracted from a fit, using a Hertz-like model, taking into account the shape of the indentor. As an example, for a pyramidal, square-based indentor of half angle to the faces $\alpha$, the relation between force, $F$, and indentation, $\delta$ follows $F=0.7453 E \tan (\alpha) \delta^{2} /\left(1-v^{2}\right) \quad\left({ }^{33}\right.$, as used in the JPK-DP processing software (JPK Instruments). The Poisson ratio, $v$, is usually set to 0.5 , which means that the cell is considered as non compressible. ${ }^{\text {xvi }}$

The indentation is $\left(h-h_{0}\right)-\left(z-z_{0}\right)$ where $h$ is the piezo displacement and $z$ the deflection of the lever. « 0 » indexes represent the offsets in height and force. This is equivalent to the tip-sample separation as presented on Fig. 2. Care has to be given to find the correct position of the contact point that is the point where the tip touches the cell, ie. where the force starts to be non null ${ }^{34}$.

Moreover, E can vary with the pressing speed, as an indication of the visco-elasticity of the cell, so experiments (and controls) have to be performed keeping this parameter constant as much as possible.

Typical values of $E$ for eukaryotic cells are found in the range $10 \mathrm{~Pa}-10 \mathrm{kPa}$.

A schematics of the principal steps of processing the pushing curve is presented on Fig. 2. They can be either included in homemade programs (eg. in Python, R, Igro Pro, C/C++ or Matlab) or used through AFM manufacturer software solutions (eg. here, JPK-DP software).

\section{Membrane tension}

Membrane tension can be accessed through the force needed to extract a tube from the cell. As exemplified on Fig. 3, a membrane tube pulling event is characterized by the presence of a plateau preceeding the rupture event (corresponding to the rupture / detachment of the tube from the tip of the lever). The measurement of the length (often set as $>100 \mathrm{~nm}$ for a tube) and slope of the plateau ( $\sim 0$ $\mathrm{pN} / \mu \mathrm{m}$ for a tube), together with the plateau force, can be done using homemade programs (eg. in Python, R, Igro Pro, C/C++ or Matlab) or through AFM manufacturer software solutions (eg. JPK-DP software). 
Care has to be given to use only force curves where one force step is present or to use the last force step having the right characteristics to ensure that the recorded forces are corresponding to tube pulling ${ }^{35}$. Usually, the fraction of curves where a tube is observed is kept below $30 \%$ to do so.

The force of the pulling event itself represents a direct evaluation of the tension. If a tension value is needed, one can convert this force, $f$, to an apparent tension, $T$, by using the approximation $f=2 \pi(2 \mathrm{~T} \kappa)^{1 / 2}$ where $\kappa$ is the membrane bending rigidity $\left(\sim 2-3 \times 10^{-19} \mathrm{Nm}\right){ }^{36}$. Depending on the variation of $f$ as a function of the pulling speed (see note xiii), more complex models can be applied 20.

Aside, $T$ calculated using the previous way on unperturbed cells is an apparent tension since it contains the contributions of the membrane, $\sigma$, and of the anchorage of the membrane to the cytoskeleton, $W_{0}$. A simple approach is to consider that $T=\sigma+W_{0}$, and obtain values of $\sigma$ by performing experiments where the membrane / cytoskeleton interactions are removed (ie. where $W_{0}=0 \quad$ ). ${ }^{20}$. Typical values of $f$, resp. $T$, for eukaryotic cells are found in the range 10-100pN, resp. $10-100 \mu N / m$.

\section{Statistical representation \& tests}

In order to present the data, several options are available, depending on what is the source of variation that one wants to investigate. The simplest way is to have, per condition, one scatter plot of the tether force or tension. Then, depending on the shape of the point cloud and its symetry, one can choose to present central tendencies by using whisker-plot (for non gaussian data) or mean +/- SD (or SEM) (for gaussian data). This representation guides the statistical tests that have to performed to compare samples, eg. for two data sets (positive vs. control), either Wilcoxon or Student test, respectively. If the data is paired (eg. Same cell tested under two conditions), this can lead to a « before / after » plot, where the tendency over a population is seen through direct links between individuals. Tests have to be modified accordingly to the data pairing. Some softwares may guide the user through the choice of the proper test (eg. GraphPad Prism, or BioStaTGV - an online application ${ }^{37}$ ). Representations and tests can be standardized and automatized by using scripting langages (eg. R, Python, IgorPro, MatLab).

The previous presentation hides in fact one complexity : what does each point represents ? One tether ? The average over all tethers measured on one cell ? The average over the cells of a given sample ? This is let to the choice of the experimentalist but has to be specified in order to clarify from where the 
measurements dispersion is coming from and what is represented by the data : cell type (here MDA vs. BT cells) or condition (BT with cFasL vs. BT cells with pcDNA3). Choosing to represent the average tension per cell, we observed a slight decrease of the mean apparent tension when cl-CD95L was incubated at $100 \mathrm{ng} / \mathrm{mL}$ with MDA cells as compared to pcDNA3. The same behaviour was observed for BT cells (PHP, unpublished data).

Aside of these classical «1D » representation, some authors proposed to use more complex, « $2 \mathrm{D}$ » maps of tether force vs. tether length to visualize subpopulations of events ${ }^{35}$ 


\section{Figures and captions}

Figure 1 : Using AFM force curves to investigate cell mechanics. A Top view micrograph of a gold less cantilever tip positioned above the nucleus zone of a fibroblast cell that is adhered to a glass bottom petri dish. The pyramidal tip can be seen. B, C Schematics of the experiments, in side view, with respectively, pressing on the cell to indent it and pulling on the membrane to extract a nanotube. D, E Typical couple of force curves, light red for B (ie. pressing) and dark red for C (ie. pulling), obtained successively. Note that $\mathrm{D}$ and $\mathrm{E}$ are presenting the same curves, the only difference being that we highlighted, on D, the fitted zone for extracting elastic measurements (Young modulus) via the application of a Hertz-like model, and, on E, the zone where the measure of the tube extraction force is performed, which is directly linked to the membrane tension. The morphology of the curve, showing a clear step-like, is the signature of the presence of a tube.

Fig 2 : Minimal sequence of data processing for mechanical measurements using the pressing part of the AFM force curves. Note that the important step is the calculation of the tip sample separation (third graph) that corresponds to the real indentation by correcting the piezo motion from the cantilever deflection. One has to be careful to check that the determination of the contact point, when fitting, is correct since errors in the Young values. Greyed zones are the selections for an automatic detection of the baseline and application of the Hertz/Sneddon fit by the JPK-DP software. Typical Young modulus for cells depend on spreading state, cell type and health. They are found, for the present cells, in the range $100 \mathrm{~Pa}-1 \mathrm{kPa}$

Fig 3 : Typical pulling parts of the AFM force curves. Only unfrequently tubes are pulled (20-30\% of the force curves). The main population of force curves exhibit either no interaction or adhesive events. Those latter have a non null slope before the force jump. On the last graph the presence of an adhesive jump before the pulling of the tube can be seen, that translates into a large and clear force plateau. In green, average curves by using a running average box of 20 points. In grey, linear fit of the force curve in the region before the force jump, showing a high slope for adhesive events and a null one for tether pulling. Typical forces for tube pulling are found for the present cells between 25 and $75 \mathrm{pN}$, resulting in apparent tensions between 20 and $400 \mu \mathrm{N} / \mathrm{m}$. Greyed zones are the selections for an automatic detection of the force jumps and slopes by the JPK-DP software. 


\section{Aknowledgments}

Funding : PhysCancer « H+-cancer » (to L. Counillon \& PHP). InCa PLBiol 2014 (to P. Legembre \& PHP). Labex INFORM (ANR-11-LABX-0054) and A*MIDEX project (ANR-11-IDEX-0001-02), funded by the « Investissements d’Avenir » French Government program managed by the French National Research Agency (ANR) (to Inserm U1067 Lab and as PhD grant to AS).

Providing material or technical help : M. Biarnes-Pelicot (U1067, Marseille), L. Counillon (Nice), A. Fouqué \& P. Legembre (Rennes).

Discussions and comments on the manuscript : L. Limozin (U1067, Marseille), L. Counillon (Univ. Nice) \& P. Legembre (Rennes).

Platforms : cell culture platform “PCC” (Luminy Campus, Marseille) - L. Borge

Companies : JPK Instruments (Berlin, Germany) for continuous support and generous help. 


\section{References}

1. Fouqué, A., Debure, L. \& Legembre, P. The CD95/CD95L signaling pathway: A role in carcinogenesis. Biochim. Biophys. Acta BBA - Rev. Cancer 1846, 130-141 (2014).

2. Tauzin, S. et al. The naturally processed CD95L elicits a c-yes/calcium/PI3K-driven cell migration pathway. PLoS Biol. 9, e1001090 (2011).

3. Edmond, V. et al. Downregulation of ceramide synthase-6 during epithelial-to-mesenchymal transition reduces plasma membrane fluidity and cancer cell motility. Oncogene 34, 996-1005 (2015).

4. Sok, M., Sentjurc, M., Schara, M., Stare, J. \& Rott, T. Cell membrane fluidity and prognosis of lung cancer. Ann. Thorac. Surg. 73, 1567-1571 (2002).

5. Billaudeau, C. et al. Probing the plasma membrane organization in living cells by spot variation fluorescence correlation spectroscopy. Methods Enzymol. 519, 277-302 (2013).

6. Picas, L., Rico, F., Deforet, M. \& Scheuring, S. Structural and mechanical heterogeneity of the erythrocyte membrane reveals hallmarks of membrane stability. ACS Nano 7, 1054-1063 (2013).

7. Gauthier, N. C., Masters, T. A. \& Sheetz, M. P. Mechanical feedback between membrane tension and dynamics. Trends Cell Biol. 22, 527-535 (2012).

8. Masters, T. A., Pontes, B., Viasnoff, V., Li, Y. \& Gauthier, N. C. Plasma membrane tension orchestrates membrane trafficking, cytoskeletal remodeling, and biochemical signaling during phagocytosis. Proc. Natl. Acad. Sci. U. S. A. 110, 11875-11880 (2013).

9. Schneider, D. et al. Tension monitoring during epithelial-to-mesenchymal transition links the switch of phenotype to expression of moesin and cadherins in NMuMG cells. PloS One 8, e80068 (2013).

10. Binnig, G., Quate, C. F. \& Gerber, C. Atomic Force Microscope. Phys. Rev. Lett. 56, 930-933 (1986).

11. Franz, C. M. \& Puech, P.-H. Atomic Force Microscopy: A Versatile Tool for Studying Cell Morphology, Adhesion and Mechanics. Cell. Mol. Bioeng. 1, 289-300 (2008).

12. Helenius, J., Heisenberg, C.-P., Gaub, H. E. \& Muller, D. J. Single-cell force spectroscopy. J. Cell Sci. 121, 1785-1791 (2008).

13. Dufrêne, Y. F. et al. Five challenges to bringing single-molecule force spectroscopy into living cells. Nat. Methods 8, 
123-127 (2011).

14. Richelme, F., Benoliel, A. M. \& Bongrand, P. Dynamic study of cell mechanical and structural responses to rapid changes of calcium level. Cell Motil. Cytoskeleton 45, 93-105 (2000).

15. Guillou, L., Babataheri, A., Puech, P.-H., Barakat, A. I. \& Husson, J. Dynamic monitoring of cell mechanical properties using profile microindentation. Sci. Rep. 6, 21529 (2016).

16. Bufi, N. et al. Human Primary Immune Cells Exhibit Distinct Mechanical Properties that Are Modified by Inflammation. Biophys. J. 108, 2181-2190 (2015).

17. Rigato, A., Rico, F., Eghiaian, F., Piel, M. \& Scheuring, S. Atomic Force Microscopy Mechanical Mapping of Micropatterned Cells Shows Adhesion Geometry-Dependent Mechanical Response on Local and Global Scales. ACS Nano 9, 5846-5856 (2015).

18. Krieg, M. et al. Tensile forces govern germ-layer organization in zebrafish. Nat. Cell Biol. 10, 429-436 (2008).

19. Krieg, M., Dunn, A. R. \& Goodman, M. B. Mechanical control of the sense of touch by $\beta$-spectrin. Nat. Cell Biol. 16, 224-233 (2014).

20. Diz-Muñoz, A. et al. Control of directed cell migration in vivo by membrane-to-cortex attachment. PLoS Biol. 8, e1000544 (2010)

21. Wu, H. W., Kuhn, T. \& Moy, V. T. Mechanical properties of L929 cells measured by atomic force microscopy: effects of anticytoskeletal drugs and membrane crosslinking. Scanning 20, 389-397 (1998).

22. Alcaraz, J. et al. Microrheology of human lung epithelial cells measured by atomic force microscopy. Biophys. J. 84, 2071-2079 (2003).

23. Eghiaian, F., Rigato, A. \& Scheuring, S. Structural, mechanical, and dynamical variability of the actin cortex in living cells. Biophys. J. 108, 1330-1340 (2015).

24. Cazaux, S. et al. Synchronizing atomic force microscopy force mode and fluorescence microscopy in real time for immune cell stimulation and activation studies. Ultramicroscopy 160, 168-181 (2016).

25. MDA-MB-231 ATCC ${ }^{\circledR}$ HTB-26 ${ }^{\mathrm{TM}}$ Homo sapiens mammary gland/breast. Available at: http://www.lgcstandardsatcc.org/Products/All/HTB-26.aspx. (Accessed: 5th April 2016)

26. BT-549 ATCC ® HTB-122 ${ }^{\mathrm{TM}}$ Homo sapiens mammary gland; breast d. Available at: http://www.lgcstandardsatcc.org/products/all/HTB-122.aspx?geo_country=fr. (Accessed: 5th April 2016) 
27. Puech, P.-H. et al. Measuring cell adhesion forces of primary gastrulating cells from zebrafish using atomic force microscopy. J. Cell Sci. 118, 4199-4206 (2005).

28. Ulrich, F. et al. Wnt11 functions in gastrulation by controlling cell cohesion through Rab5c and E-cadherin. Dev. Cell 9, 555-564 (2005).

29. Evans, E. \& Kinoshita, K. Using force to probe single-molecule receptor-cytoskeletal anchoring beneath the surface of a living cell. Methods Cell Biol. 83, 373-396 (2007).

30. Butt, H.-J. \& Jaschke, M. Calculation of thermal noise in atomic force microscopy. Nanotechnology 6, 1 (1995).

31. Merkel, R., Nassoy, P., Leung, A., Ritchie, K. \& Evans, E. Energy landscapes of receptor-ligand bonds explored with dynamic force spectroscopy. Nature 397, 50-53 (1999).

32. Brochard-Wyart, F., Borghi, N., Cuvelier, D. \& Nassoy, P. Hydrodynamic narrowing of tubes extruded from cells. Proc. Natl. Acad. Sci. U. S. A. 103, 7660-7663 (2006).

33. Bilodeau, G. Regular Pyramid Punch Problem. J. Appl. Mech.-Trans. Asme 59, 519-523 (1992).

34. Crick, S. L. \& Yin, F. C.-P. Assessing micromechanical properties of cells with atomic force microscopy: importance of the contact point. Biomech. Model. Mechanobiol. 6, 199-210 (2007).

35. Sariisik, E. et al. Decoding Cytoskeleton-Anchored and Non-Anchored Receptors from Single-Cell Adhesion Force Data. Biophys. J. 109, 1330-1333 (2015).

36. Hochmuth, F. M., Shao, J. Y., Dai, J. \& Sheetz, M. P. Deformation and flow of membrane into tethers extracted from neuronal growth cones. Biophys. J. 70, 358-69 (1996).

37. BiostaTGV - Statistiques en ligne. Available at: http://marne.u707.jussieu.fr/biostatgv/. (Accessed: 30th March 2016) 


\section{Figure 1}

A

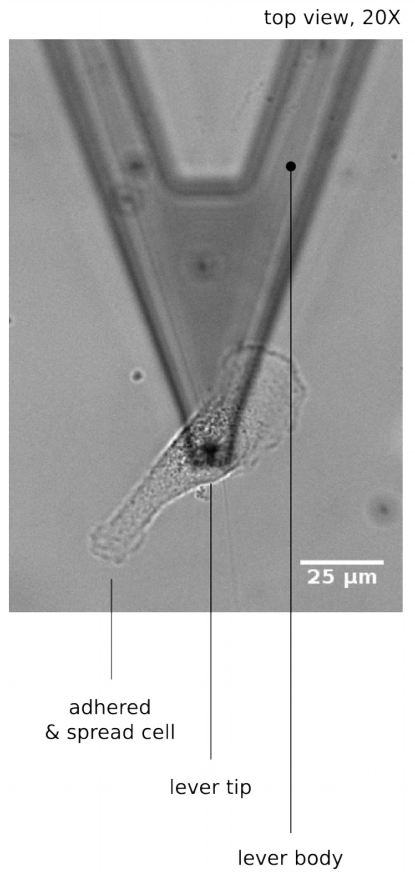

B

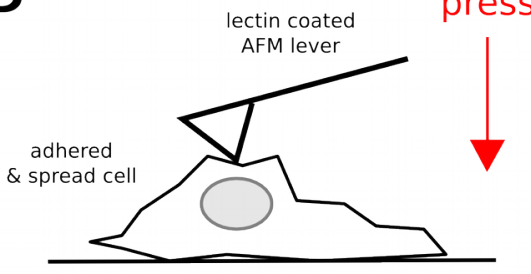

D

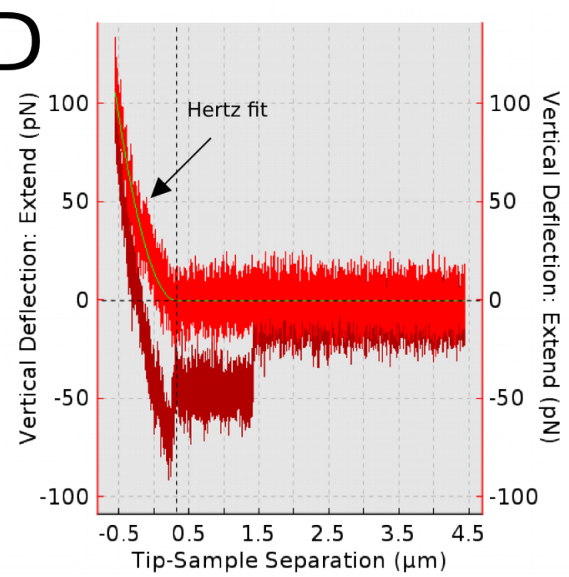

C
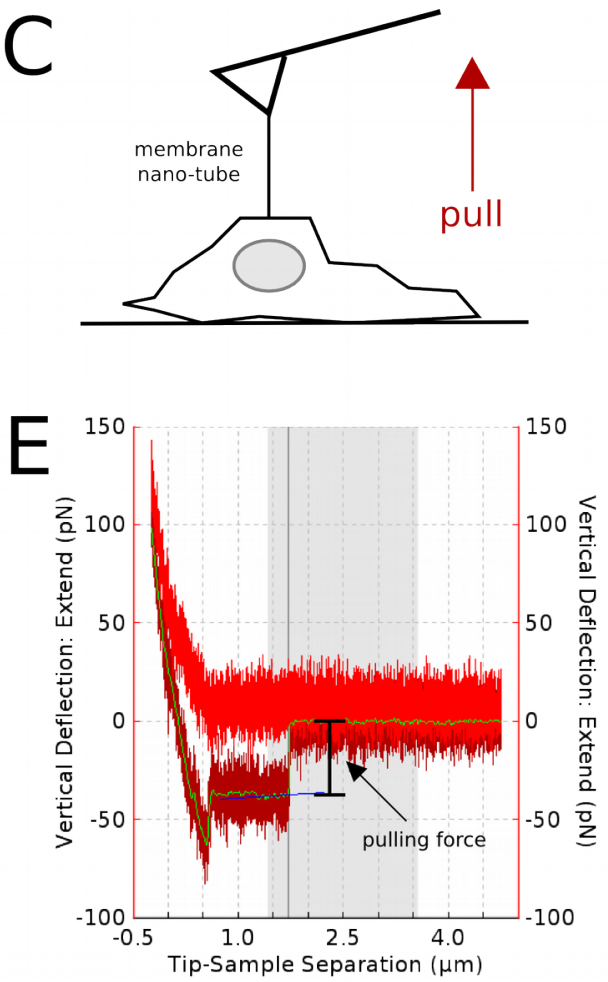
Figure 2
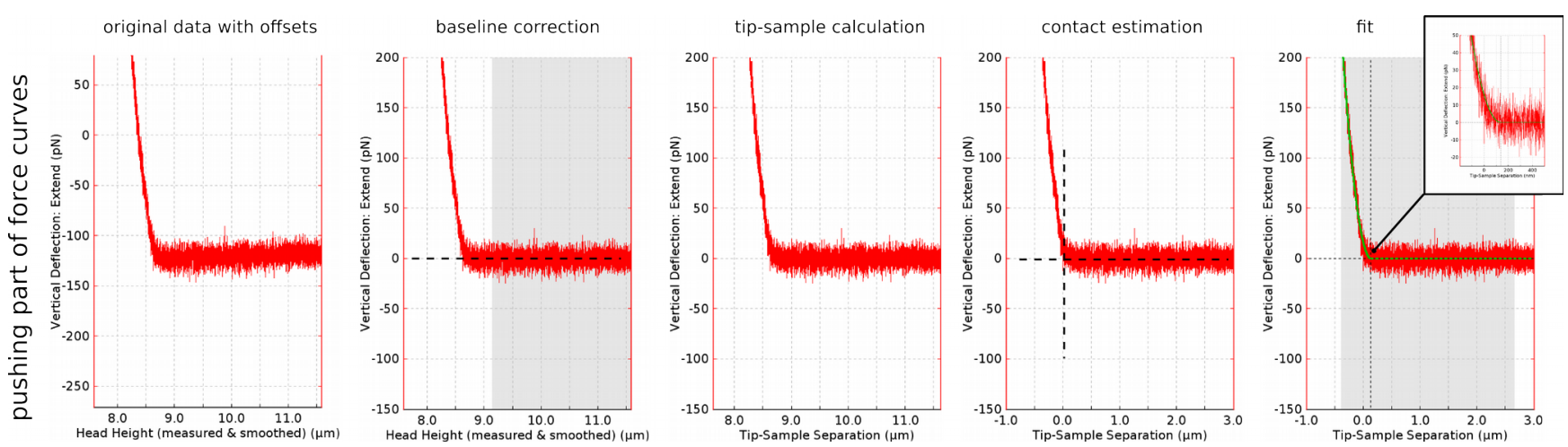
Figure 3
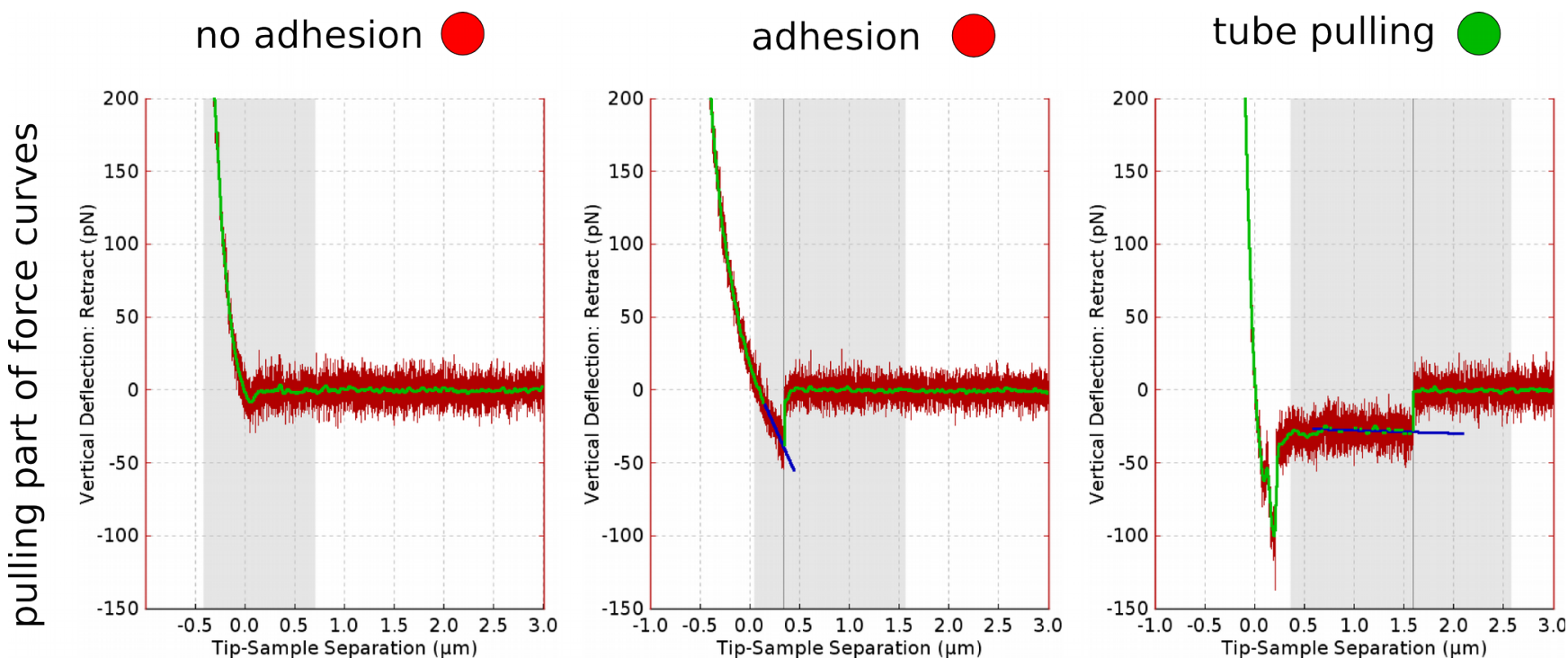


\section{Notes}

i The present protocol has been performed using a JPK Nanowizard I (JPK Instrument, Germany) mounted on an Axiovert 200 inverted microscope (Zeiss), equipped with 10x, 20x and 40x lenses, coupled to a Colibri 2 diodes system (Zeiss) with suitable filter sets for fluoresence microscopy ${ }^{24}$. This protocole may require some adaptation to be used with other AFM brands, but stays globally the same as long as the AFM sits on an inverted microscope.

ii Another type of lever that can be used is MicroBiolever from Olympus $(6 \mathrm{pN} / \mathrm{nm})^{19}$.

iii Mycoplasm absence was verified once a month using culture supernatants following a procedure of the cell culture platform (Mycoalert, Lonza).

iv Cell concentration and seeding volume were adjusted so that cells are mainly found, after 24hrs of culture, separated one from the other, in order to allow for single cell measurement.

$\mathrm{v}$ Here, the experiments are performed at room temperature. Temperature can be set and / or varied using adapted equipments such as PetriDish Holder (JPK Instruments) or homemade environmental chambers.

vi Gold-less cantilevers are more stable over time (the force baseline is better) and they are more transparent than regular levers ${ }^{24}$. The latter point eases the positionning the very tip of the lever onto a given part of a cell (see figure : the pyramidal tip is the darker point at the extremity of the $\mathrm{V}$ shape lever)

vii Variation of the proposed protocol involves the adsorption of a first biotinylated BSA layer, then streptavidin and finally, the coupling or a biotinylated lectin. This ensures that the lectin is less likely to desorb over the time course of the experiment and that it is preferentially oriented (see $11,18,27,28)$.

viii The molecule used to decorate the tip depends on the choice of the experimentalist to pull in a non differenciated manner on the membrane by "fishing » any sugar residues using lectins rather than pulling on specific linker eg. by using a dedicated antibody or hapten (fibronectin or RGD, or collagen, peptide to target certain classes of integrin, ...). For an example of specific tube pulling, see ${ }^{29}$.

ix Do not forget to center it properly. This ensure that the relative motion of the lever and the sample will be large and easy, and also it helps to rapidely focus the laser on the lever back from experiment to experiment, by keeping an alsmost constant lever position.

$\mathrm{x}$ Be careful not to block the laser light by using inappropriate fluorescence filters. For the JPK Instrument set-up, the laser is infrared, so it can be detected by a CCD camera when no filter is present.

xi Do not let the lever interact for a long time with the substrate in order to keep its coating intact .

xii Tip to cell interactions may be modulated by the presence of divalent cations in the culture medium. Sometimes, the presence of a low level of albumin or serum may also help to modulate the interaction, by supressing non specific interactions.

xiii It may be needed to check rapidely that the calibration parameters did not change when moving the lever out of the sample. In particular, the sum signal should not change.

xiv It has been long shown that molecular rupture forces are dependant on the local loading rate ${ }^{31}$, so retraction speed has to be kept constant. It can be varied to do more complex measurements ${ }^{19,20,32}$

$\mathrm{xv}$ The lever «efficiency» for pulling tube may decrease over time and force to slightly adapt the force and time parameters of the experiments. This may due to the loss of antibody when only adsorbed at the lever tip surface, where their number is small due to the reduced size of the tip's end. Another possibility that may account for this disminished efficiency is the potential pollution of the tip by cell's material or debris eg. residues of membrane. To reduce this incertainty, in particular in the case of taking numerous ( $>25)$ force curves per cell, we recommend to limit both the number of curves per cell and the number of cells per experiment, to respectively, typically 10 per cell and 15 per sample, and to increase statistics by multiplying the number of separate experiments (separate sample and separate lever), taking into account the variability coming from lever to lever as well as sample to sample.

xvi This model can be considered as valid here since the adhesion is kept at low level and rather unfrequent. If adhesion is large, other physical models, taking into account the fact that the work of adhesion may participate in the deformation, such as JKR or DMT models, have to be considered. 